\title{
Disposición del alumnado al uso de herramientas de comunicación síncrona en la docencia universitaria
}

Francisco Grimaldoa ${ }^{a}$ Emilia López-Iñestab, David Arnauc, Máximo Cobos-Serrano ${ }^{a}$, Ricardo Ferrís ${ }^{a}$, Miguel García-Pineda ${ }^{a}$, Juan Gutiérrez-Soto ${ }^{c}$, Paloma Moreno-Claría, Mario Zacarés-González ${ }^{\mathrm{b}}$ y Carlos Pérez-Conde ${ }^{\mathrm{a}}$

a'Departament d'Informàtica, Universitat de València, Av. De la Universitat s/n, 46100, Burjassot, España, francisco.grimaldo@uv.es, Maximo.Cobos@uv.es, ricardo.ferris@uv.es, Miguel.GarciaPineda@uv.es, paloma.moreno@uv.es, carlos.perez@uv.es, bFacultad de Ciencias de la Actividad Física y del Deporte, Universidad Católica de Valencia "San Vicente Mártir”, Avda. Virgen de la

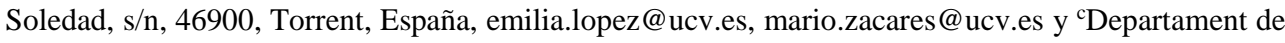
Didàctica de la Matemàtica, Universitat de València, 46022, València, España, david.arnau@uv.es, juan.gutierrez-soto@uv.es.

\begin{abstract}
In recent years there have been numerous innovative educational initiatives that have proposed the use of synchronous communication tools to support university teaching. These proposals have proven their worth in specific application contexts such as: virtual tutoring, internationalization and mobility in masters and doctorates. However, their sometimes limited scope often makes it difficult to generalize results to other contexts and, thus, to pass from pilots to consolidated solutions. Within the framework of 2015 being declared as the International Year of Evaluation, this paper presents the results of a survey aimed to obtain the opinion of students regarding the use of synchronous communication tools for university teaching. The survey involved 436 students enrolled in subjects of various grade levels in degrees sucha as engineering, teaching, criminology and sports science, both in public and private universities. The results show a characterization of the interest, expectations and preferences of students with respect to the incorporation of computer communication tools as a mechanism to improve the learning process.
\end{abstract}

Keywords: synchronous communication tool, students opinon, higher education 
Disposición del alumnado al uso de herramientas de comunicación síncrona en la docencia universitaria

\begin{abstract}
Resumen
En los últimos años han sido numerosas las iniciativas de innovación educativa que han propuesto el uso de las herramientas de comunicación síncrona como recurso tecnológico de soporte a la docencia universitaria. Dichas propuestas han demostrado sus beneficios en contextos de aplicación concretos como por ejemplo: la tutorización virtual o la internacionalización y movilidad en másteres y doctorados. Sin embargo, su alcance limitado dificulta a menudo la extrapolación de resultados a otros ámbitos docentes y, por tanto, la transformación de actividades que se pueden catalogar como piloto en soluciones de implantación consolidada. Con el trasfondo de la declaración del año 2015 como Año Internacional de la Evaluación, en este trabajo presentamos los resultados de un estudio dirigido a conocer la opinión del alumnado sobre el uso de herramientas de comunicación por Internet en la docencia universitaria. El estudio ha involucrado a 436 estudiantes matriculados en asignaturas de cursos diversos en titulaciones de ingeniería, magisterio, criminología y ciencias del deporte, en universidades públicas y privadas. Los resultados muestran una caracterización del interés, las expectativas y las preferencias de los alumnos con respecto a la incorporación de las herramientas informáticas de comunicación síncronas como mecanismo de apoyo y mejora del proceso de aprendizaje.
\end{abstract}

Palabras clave: herramientas de comunicación síncrona, opinión alumnado, educación superior.

\title{
1. Introducción
}

El año 2015 ha sido declarado el Año Internacional de la Evaluación con el fin de reemplazar a los Objetivos de Desarrollo del Milenio y promover la elaboración de Objetivos de Desarrollo Sostenible. Como punto de partida se pretende una evaluación de lo que se ha conseguido hasta el momento en distintos niveles de la sociedad civil entre los que figura la educación.

La preocupación por la mejora en la calidad de la docencia universitaria tiene un objetivo, en apariencia principal, que es la propia formación de los universitarios y un segundo objetivo, central, ligado a la repercusión de esta formación en la sociedad. Es por ello que esta exigencia es particularmente mayor en aquellas titulaciones de carácter profesionalizador, lo que ya ha dado lugar a estudios para evaluar la calidad de la formación universitaria recibida. Por ejemplo, entre 2007 y 2008 se recogieron los datos del Teacher Education and Development Study in Mathematics (TEDS-M) organizado por la International Association for the Evaluation of Educational Achievement. El objetivo de este estudio era analizar la 
Francisco Grimaldo, Emilia López-Iñesta, David Arnau, Máximo Cobos-Serrano, Ricardo Ferrís, Miguel García-Pineda, Juan Gutiérrez-Soto, Paloma Moreno-Clarí, Mario Zacarés-González y

Carlos Pérez-Condes

formación inicial en matemáticas y didáctica de la matemática de los estudiantes de titulaciones universitarias que permitían impartir docencia de matemáticas en primaria y secundaria. Más allá de las limitaciones del estudio (véase, por ejemplo, Döhrmann, Kaiser y Blömeke, 2014), los resultados situaron a los estudiantes universitarios españoles por debajo de la media respecto al resto de países que participaron en el estudio (Gómez y Gutiérrez-Gutiérrez, 2014). De estos resultados podía deducirse una crítica a la formación recibida y la necesidad de mejorar la docencia.

Una de las formas de mejorar la docencia presencial es recurriendo a formas de enseñanza típicas de la formación a distancia que permiten una interacción entre estudiantes y entre estudiantes y profesores más inmediata. La aparición de herramientas de comunicación específicamente diseñadas para la docencia ha revolucionado estas técnicas. Por otro lado, la exigencia de la declaración de Bolonia de introducir metodologías de enseñanza que fomenten la cooperación y de promover la dimensión europea de la educación superior han llevado a la necesidad de contemplar y dar solución a nuevos escenarios de interacción en la docencia presencial (véase, por ejemplo, Grimaldo-Moreno, Arevalillo-Hérraez y LópezIñesta, 2011).

Numerosas investigaciones han descrito las ventajas e inconvenientes de las herramientas de comunicación síncronas y asíncronas en escenarios educativos. Así, Johnson (2008) concluyó que las discusiones asíncronas dan tiempo para pensar las respuestas y que, en consecuencia, fuerzan a los estudiantes a prepararse. Sin embargo, las discusiones síncronas permiten obtener más información y de manera más rápida. En la misma línea, Sancerni (2012) indica que más de la mitad de los estudiantes universitarios que participaron en un estudio prefirieron realizar tutorias síncronas virtuales a tener que realizarlas presencialmente, pero más del $90 \%$ las consideró sólo útiles para consultas pequeñas. Por otro lado, uno de los factores que influyen en el éxito en el uso de herramientas asíncronas es el carácter obligatorio u opcional de su uso. Wang (2004) observó que los estudiantes que se hacían más visibles en las discusiones superaban a sus compañeros en las puntuaciones de la asignatura. Evidentemente, esto podría ser una consecuencia de la capacidad cognitiva de los participantes y no tanto del tipo de participación. De hecho, en Jonhson (2005) se constata que una escasa participación predice bajos resultados académicos, pero una alta participación no necesariamente implica mejores resultados. Por otro lado, el uso de herramientas síncronas permite soslayar dificultades propias de la interacción cara a cara (Lobel, Neubauer, y Swedburg, 2002), pero exige un mayor protagonismo del tutor para dirigir los procesos de comunicación (Burnett, 2003; Hsieha y Tsai, 2012). De hecho, Offir, Lev, y Bezalel (2008) señalan que el nivel de interacción entre profesor y estudiantes es uno de los factores que determinan la eficacia de un método de enseñanza y concluyen que los estudiantes prefieren sistemas de comunicación síncronos a asíncronos cuando los usan con intenciones

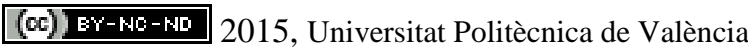

Congreso In-Red (2015) 
Disposición del alumnado al uso de herramientas de comunicación síncrona en la docencia universitaria

educativas. En este estudio también se puso de manifiesto que las técnicas síncronas son más efectivas en estudiantes con habilidades cognitivas altas, mientras que en el caso de estudiantes con habilidades cognitivas bajas parecía eran más adecuadas las técnicas asíncronas.

Así pues, en este contexto de evaluación de las propias técnicas de innovación docente, el presente trabajo presenta un estudio sobre la disposición del alumnado al uso de herramientas de comunicación síncrona en la docencia universitaria que tiene en cuenta un abanico de asignaturas, cursos y grados de universidades públicas y privadas.

\section{Objetivos}

Las herramientas de comunicación síncrona son, en la actualidad, un elemento clave en los procesos de enseñanza-aprendizaje (Díaz et al., 2014), como se indica en la sección anterior. Este tipo de herramientas se están usando en diversos ámbitos de la educación, siendo una referencia en el ámbito de la formación específica a distancia. No obstante esto está cambiando y, cada vez más, se están implantando en otro tipo de ámbitos como puede ser la docencia universitaria presencial. Pero, ¿conocen los alumnos universitarios este tipo de herramientas síncronas?, ¿saben exactamente qué se puede realizar con ellas?, ¿todos los alumnos tienen el mismo interés por hacer uso de ellas en el proceso de enseñanzaaprendizaje en el ámbito universitario?, ¿cúal sería la curva de aprendizaje para hacer un uso adecuado de las mismas?

Todas estos aspectos y muchos más se pretenden analizar en este trabajo, cuyo objetivo principal es el diseño e implementación de un cuestionario que permita recopilar y conocer la opinión del alumnado sobre el empleo de herramientas de comunicación síncrona como complemento a la docencia universitaria presencial y, más concretamente, si consideran que su uso puede ayudarles significativamente en el proceso de enseñanza-aprendizaje.

Además, este trabajo tiene como objetivos adicionales:

- Analizar si existen diferencias de interés en función de la titulación elegida y, especialmente, si estas diferencias dependen de si la titulación tiene o no un marcado carácter técnico.

- Estudiar si las opiniones varían dependiendo de si se trata de centros públicos o privados o de si se trata de estudiantes de primero o de cursos más avanzados.

- Averiguar qué herramientas prefieren en caso de que ya las utilicen y, en cualquier caso, averiguar qué herramientas son más conocidas (por ejemplo, BLACKBOARD COLLABORATE, BIGBLUEBUTTON, ADOBE CONNECT, GOOGLE HANGOUTS, etc.). 
Francisco Grimaldo, Emilia López-Iñesta, David Arnau, Máximo Cobos-Serrano, Ricardo Ferrís, Miguel García-Pineda, Juan Gutiérrez-Soto, Paloma Moreno-Clarí, Mario Zacarés-González y

Carlos Pérez-Condes

\section{Desarrollo de la innovación}

Este apartado muestra los detalles del estudio de disposición del alumnado al uso de herramientas de comunicación síncrona en la docencia universitaria. El estudio involucró a 436 estudiantes de grado, matriculados en asignaturas de los tres primeros cursos académicos, en titulaciones del área de la ingeniería, magisterio, criminología y ciencias del deporte. Los alumnos provenían de la Universitat de València (UV) y de la Universidad Católica de Valencia (UCV), siendo la primera una institución pública y la segunda privada.

\subsection{Diseño del cuestionario}

El estudio que se expone en este trabajo está basado en las respuestas obtenidas a través de un cuestionario cuidadosamente diseñado para reflejar la opinión de los alumnos sobre el uso de herramientas de comunicación por Internet en diferentes aspectos.

El cuestionario se llevó a cabo siguiendo el método Delphi (Linstone y Turoff, 1975). Este método permite llegar a un cuestionario consensuado de forma sistemática, recopilando la opinión de un panel de expertos e iterando sobre las valoraciones ofrecidas por los mismos hasta alcanzar el consenso. Los propios autores de este estudio actuaron como panel de expertos, siendo uno de ellos el moderador.

Con este fin, el método se aplicó en dos etapas. En la primera de ellas, las rondas de respuestas se orientaron a decidir los apartados que debía contener el cuestionario para poder definir apropiadamente los diferentes perfiles que pudieran surgir del análisis de las respuestas por parte de los alumnos. En la segunda, se decidieron las preguntas a incluir en cada uno de los apartados. En cualquier caso y antes de comenzar el diseño del cuestionario, se decidió que el cuestionario debía contener un conjunto de preguntas relativamente reducido, debía poder ser comprendido con facilidad por parte del alumnado y debía evitar posibles ambigüedades y/o interpretaciones alternativas. De la misma forma, se estableció con anterioridad el objetivo del estudio: "Conocer la valoración que los alumnos de diferente perfil académico dan a la utilidad de incluir herramientas de comunicación por Internet en la docencia universitaria”.

Los apartados consensuados incluidos en el cuestionario son los siguientes:

- Información personal y académica: pretende recoger información del alumno referente a su edad y género, universidad, titulación, acceso a los estudios y la situación académica en la que se encuentra.

- Manejo de herramientas informáticas: recoge información sobre la relación general que el alumno tiene con la tecnología.

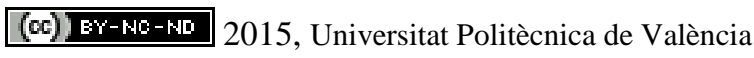

Congreso In-Red (2015) 
Disposición del alumnado al uso de herramientas de comunicación síncrona en la docencia universitaria

- Frecuencia de uso de herramientas para la comunicación por Internet: pretende recopilar información sobre el uso que hace el participante sobre herramientas de comunicación síncrona en su uso personal y académico, así como su conocimiento acerca de algunas plataformas concretas.

- Nombre de herramientas de comunicación por internet que usa con más frecuencia: se trata de un ítem de respuesta libre para conocer qué herramientas utiliza principalmente el alumno. Esta pregunta permite también identificar qué tipo de herramientas entiende el alumno como "herramientas de comunicación por Internet”.

- Expectativas iniciales para el uso de herramientas de comunicación por Internet en la asignatura: recoge información acerca de la opinión que tiene el alumno acerca del uso de estas herramientas en la asignatura particular, su utilidad y su preferencia sobre las sesiones presenciales.

El cuestionario final resultante de la segunda etapa de elaboración de las preguntas que debe incluir cada apartado se puede consultar en el Anexo I.

\subsection{Implementación de cuestionarios en MOODLE y dotTLRN}

Otro aspecto que se decidió de manera consensuada entre el grupo de expertos fue el soporte en que debía realizarse el cuestionario. Aunque para asegurar una alta participación, parecía conveniente pasar los cuestionarios en papel al inicio de una clase, esta opción obligaba a recopilar los resultados manualmente para su posterior tratamiento y análisis. Las universidades implicadas en el estudio cuentan con una Plataforma de Gestión del Aprendizaje o Aula Virtual de funcionamiento asíncrono, que se utiliza de manera habitual en las asignaturas. Estas plataformas, a su vez, disponen de un módulo específico de creación de cuestionarios y encuestas, cuyos resultados son fácilmente exportables a otros formatos para el posterior tratamiento de los datos. De modo que teniendo en cuenta estos aspectos se optó finalmente por crear y activar el cuestionario en las plataformas docentes de las universidades participantes en el estudio: dotLRN para el caso de la Universitat de València (UV) y MOODLE en la Universidad Católica de Valencia (UCV).

dotLRN dispone del módulo Cuestionarios dotado de la suficiente versatilidad para realizar tanto exámenes, como autoevaluaciones o encuestas, con posibilidad de preguntas de tipo test de respuesta única o múltiple; o respuestas de texto libre. Asimismo puede configurarse de manera que las respuestas sean anónimas. Los profesores disponen del archivo comprimido con la encuesta común a todas las titulaciones y universidades, en formato compatible para su importación a dotLRN. De este modo, cada profesor, en su asignatura de Aula Virtual, importa el cuestionario, y al administrarlo decide el momento en que se activará, -cambiándole el estado-, y el tiempo en que permanecerá activo. Una vez finaliza el 
Francisco Grimaldo, Emilia López-Iñesta, David Arnau, Máximo Cobos-Serrano, Ricardo Ferrís, Miguel García-Pineda, Juan Gutiérrez-Soto, Paloma Moreno-Clarí, Mario Zacarés-González y

Carlos Pérez-Condes

tiempo de respuesta, y de nuevo desde el portal de administración de cuestionarios, visualiza y guarda los resultados de todos los cuestionarios de su asignatura en un archivo CSV (Comma Separated Values), de valores separados por comas, compatible para incluirlo en un archivo que contenga las respuestas de todas las asignaturas y universidades del estudio para el posterior análisis y tratamiento de los datos.

Cabe puntualizar que dotLRN dispone también del módulo Encuestas, que por definición ya son anónimas. Estas encuestas pueden utilizarse para testear la satisfacción de los alumnos con un tema concreto, para sondeos de opinión, etc. Sin embargo, en este módulo de encuestas sólo pueden configurarse respuestas de selección (tipo test), y los resultados no pueden exportarse en un formato reutilizable. De modo que la mayor versatilidad del módulo de cuestionarios en dotLRN, así como la utilidad de importación y exportación en diferentes formatos compatibles con otras plataformas, hizo que finalmente fuera éste el módulo utilizado para la implementación de la encuestas de estudio.

La plataforma Moodle tiene configurados tanto el módulo Cuestionarios como el módulo Encuestas. Estos módulos tienen diferentes utilidades. Mientras Cuestionarios se orienta a la realización de pruebas parciales, finales y de autoevaluación, sin posibilidad de envío anónimo, por tanto; Encuestas está orientado a la realimentación, es anónimo, permite importar las encuestas ya definidas en un fichero zip, y exportarlas en formato compatible. Las ncuestas en Moodle, por tanto, tiene la versatilidad y desarrollo requerido para su utilización en el estudio. Es, por tanto, el módulo utilizado. Los profesores disponen del archivo comprimido con la encuesta común a todas las titulaciones y universidades, en formato compatible para su importación al módulo Encuestas de Moodle. De este modo, cada profesor, en su asignatura de Aula Virtual Moodle, importa el cuestionario, decide el momento en que se activará, y el tiempo durante el cual permanecerá activo. Una vez finaliza el tiempo de respuesta, guarda los resultados de todos los cuestionarios de su asignatura en un formato compatible para incluirlo en un archivo que contenga las respuestas de todas las asignaturas y universidades del estudio para el posterior análisis y tratamiento de los datos.

\subsection{Recopilación de respuestas de alumnos}

Los cuestionarios implementados en las plataformas virtuales de las universidades participantes fueron abiertos al alumnado al comienzo de las distintas asignaturas consideradas en el estudio. Durante la primera sesión presencial de la asignatura, los profesores explicaron el objetivo del estudio a los alumnos de la asignatura con el fin de que éstos comprendieran el objeto y el alcance del mismo. No obstante, el propio cuestionario incluye en su comienzo una breve explicación del estudio, asegurando al alumno la confidencialidad de sus respuestas y su anonimato.

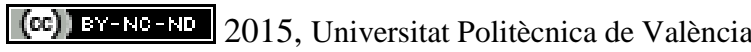

Congreso In-Red (2015) 
Disposición del alumnado al uso de herramientas de comunicación síncrona en la docencia universitaria

Si bien en un primer momento se permitió que los alumnos participaran de forma libre y rellenaran el cuestionario según su conveniencia, dada la escasa participación se decidió plantear el cuestionario como actividad a realizar en la propia clase presencial. Aunque la participación siguió teniendo un carácter voluntario, esto permitió obtener un número mucho mayor de cuestionarios completados. Contando ambas aproximaciones, el tiempo total durante el cual los cuestionarios estuvieron abiertos ascendió a 3 semanas.

\section{Resultados}

En esta sección se presenta el análisis de los datos recogidos en los cuestionarios sobre la disposición del alumnado al uso de herramientas de comunicación síncrona en la docencia universitaria. Los cuestionarios fueron contestados por 436 estudiantes de los cuales 160 estudian en la UCV y 276 en la UV, un $42 \%$ son mujeres y un 58\% son hombres, el $47.48 \%$ están matriculados en primer curso, el 31.65\% están en segundo y el 20.87\% están en tercero. Sobre el acceso a la universidad, los datos recogidos indican que el 18\% acceden al grado universitario con un título de FP, el 15\% tienen un título universitario, el 65\% han superado las Pruebas de Acceso a la Universidad y el 2\% alumnos acceden por otras vías. Con respecto a las edades, un 34\% tienen 19 o menos años, un 29\% tienen entre 20 y 21 años, un 14\% entre 22 y 23, un $8 \%$ entre 24 y 25 y un 14\% son mayores de 25 años.

La tabla 1 muestra un resumen de las asignaturas y los grados universitarios involucrados en este estudio. La columna Disciplina corresponde a una etiqueta creada para caracterizar si se trata de un grado más o menos técnico.

Tabla 1. Detalles sobre las asignaturas involucradas en el estudio

\begin{tabular}{ccccc}
\hline Asignatura - Titulación & Curso & Disciplina & Centro \\
\hline Estadística - Ciencias deporte y Act. Fís. & 1 & Deporte & UCV \\
Fundamentos Computadores - Ingeniería Informática & 1 & Ingeniería & UV \\
Informática I - Ingeniería Informática & 1 & Ingeniería & UV \\
Programación - Ingeniería Informática & 1 & Ingeniería & UV \\
Matemàtiques per a Mestres - Maestro Educ. Primaria & 2 & Humanidades & UV \\
Transmisiones, Imagen y Sonido - Derecho y Criminología & 2 & Humanidades & UV \\
Fund. Matemáticos Comunicaciones -Ing. Informática & 2 & Ingeniería & UV \\
Programación Multimedia - Ingeniería Informática & 3 & Ingeniería & UV \\
Seguridad Informática - Ingeniería Informática & 3 & Ingeniería & UV \\
Didáctica aritm. y resolución prob - Maestro Edu. Primaria & 3 & Humanidades & UV \\
\hline
\end{tabular}

Un primer aspecto que se constata para todas las disciplinas es que el concepto que el alumnado tiene de las herramientas de comunicación síncrona (figura 1) se confunde con el 
Francisco Grimaldo, Emilia López-Iñesta, David Arnau, Máximo Cobos-Serrano, Ricardo Ferrís, Miguel García-Pineda, Juan Gutiérrez-Soto, Paloma Moreno-Clarí, Mario Zacarés-González y

Carlos Pérez-Condes

de otras herramientas de comunicación no síncronas (p. ej. correo electrónico o Dropbox) o que, aunque podrían ser consideradas como de respuesta inmediata (p. ej. Whatsapp o Twitter), no disponen de funcionalidades adecuadas para la docencia (p. ej. pizarras virtuales o compartición de pantalla). Esto puede tener una incidencia negativa sobre el interés y la predisposición del alumnado a su utilización y lleva a la recomendación de que se realice una sesión demostrativa de las capacidades y potenciales usos de la herramienta seleccionada en un entorno docente, como ya han indicado estudios precedentes (Grimaldo et al., 2014).

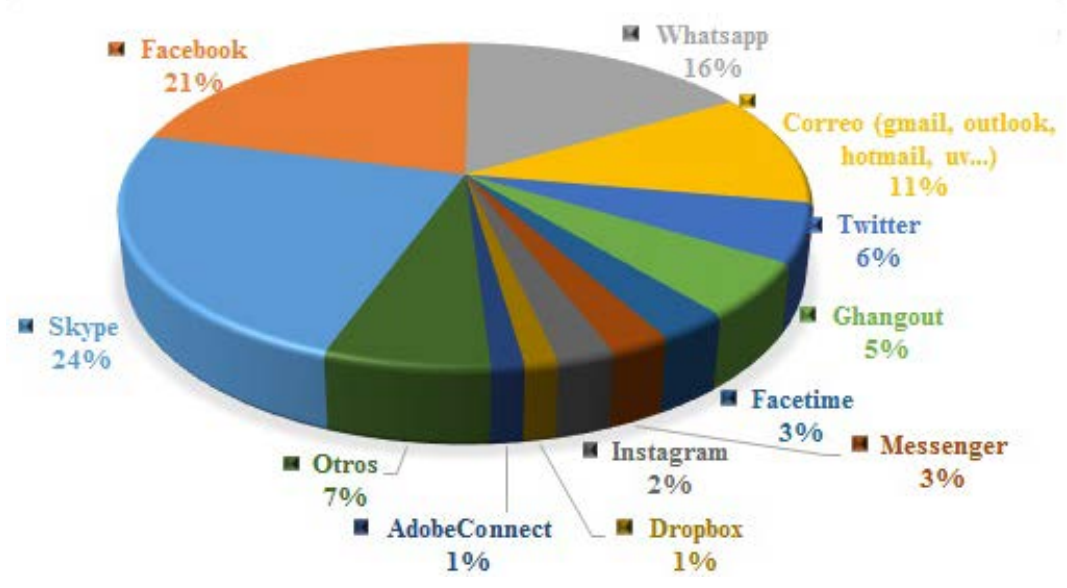

Fig. 2 Conocimiento de herramientas de comunicación síncrona por Internet

Con respecto al análisis de las variables incluidas en cada uno de los bloques del cuestionario diseñado, la mayor parte de las variables son cualitativas y por tanto esto determinará el análisis estadístico que se puede llevar a cabo. Para estudiar si existen dependencias significativas entre las variables dos a dos se han construido tablas de contingencia y se realizado tests Chi-cuadrado. Además, se empleará el análisis de correspondencias (AC) para realizar gráficos en dos dimensiones sobre los que se representarán las categorías de las dos variables. Tal y como indica Peña (2002), la mayor o menor distancia entre los puntos representados reflejan relaciones de dependencia y semejanza más o menos fuertes entre las categorías representadas. Los resultados más relevantes obtenidos son:

- Los alumnos de universidades privadas se encuentran más seguros por el hecho de disponer de herramientas síncronas para comunicarse con el profesorado (chicuadrado $=21.2799, \mathrm{df}=4, \mathrm{p}<0.0003$ ).

- Existe una relación significativa entre la variable Disciplina y la Pregunta sobre si la iniciativa de emplear las herramientas de comunicación síncronas como apoyo a

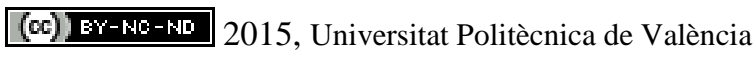

Congreso In-Red (2015) 
Disposición del alumnado al uso de herramientas de comunicación síncrona en la docencia universitaria

la docencia les parece interesante (chi-cuadrado $=27.6816$, df $=8, \mathrm{p}<0.0005$ ). Así, los alumnos de Ingeniería muestran un grado de acuerdo total cuando se les pregunta si les parece una iniciativa interesante, seguidos por los alumnos de Ciencias del Deporte y los de Humanindades. Estas relaciones se pueden observar en la figura 2 del correspondiente análisis de correspondencias, donde los valores de los ejes x e y representan los porcentajes de inercia (varianza) explicada de cada dimensión.

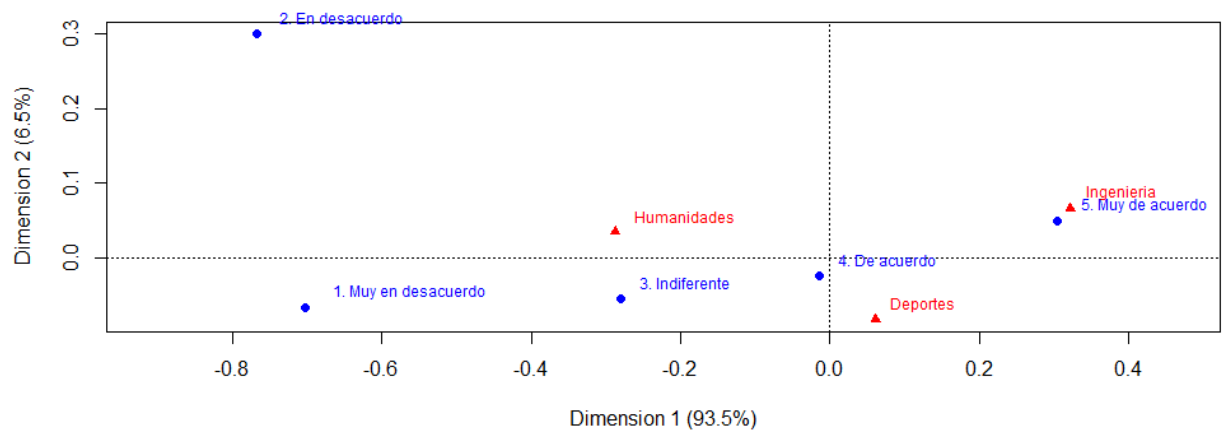

Fig. 2 Análisis de correspondencias entre la Disciplina y la pregunta Iniciativa Interesante

- El curso en el que se encuentran matriculados los alumnos tiene un efecto sobre múltiples variables. De un lado, los alumnos de primer y tercer curso muestran su interés (chi-cuadrado $=22.7406$, $\mathrm{df}=8, \mathrm{p}<0.004$ ) por el uso de herramientas síncronas como soporte al aprendizaje, mientas que los alumnos de segundo adoptan una postura indiferente o incluso se muestran en desacuerdo (ver la figura 3). La explicación a esta divergencia en la percepción se encuentra en el perfil del alumnado que: en el caso de cursar primero, afirma que las herramientas síncronas le aportan seguridad (chi-cuadrado $=38.913$, $\mathrm{df}=8, \mathrm{p}<0.005$ ); en el caso de cursar segundo, prefiere una docencia presencial y no se siente más seguro por el hecho de poder usarlas; $y$, en el caso de cursar tercero, prefiere las clases no presenciales (chicuadrado $=21.6183, \mathrm{df}=8, \mathrm{p}<0.006)$ y afirma que las herramientas síncronas le sirven para ahorrar tiempo y desplazamientos en un contexto en el que hay un mayor porcentaje de alumnos que trabajan.

- Por útlimo, el interés en una asignatura hace aumentar la percepción de utilidad de las herramientas síncronas como método de comunicación con el profesor (chicuadrado $=26.2392, \mathrm{df}=8, \mathrm{p}<0.01$ ). Sin embargo, los alumnos que no se muestran muy interesados en la asignatura tampoco lo hacen en el uso de herramientas síncronas, lo que lleva a indicar que su uso no tenga como objetivo la motivación o la lucha contra el abandono prematuro sino, más bien, la mejora de las calificaciones de los alumnos interesados. 
Francisco Grimaldo, Emilia López-Iñesta, David Arnau, Máximo Cobos-Serrano, Ricardo Ferrís, Miguel García-Pineda, Juan Gutiérrez-Soto, Paloma Moreno-Clarí, Mario Zacarés-González y

Carlos Pérez-Condes

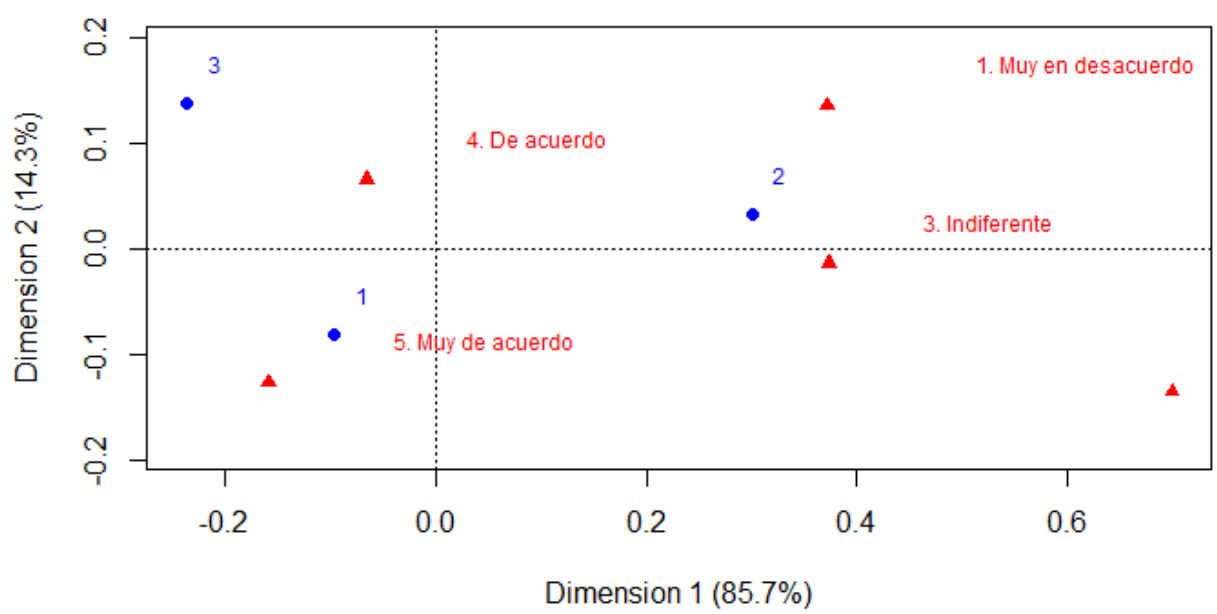

Fig. 3 Análisis de correspondencias entre el curso y la pregunta Iniciativa Interesante

\section{Conclusiones}

Son múltiples los proyectos de innovación docente que se llevan a cabo, pero en muchas ocasiones no se obtiene el resultado esperado debido a que al diseñar las propuestas de innovación no se tienen en cuenta las valoraciones y necesidades de los estudiantes. Las opiniones recogidas de los estudiantes respecto al interés y las expectativas con respecto a la participación en iniciativas docentes basadas en el uso de herramientas por Internet demuestran que continua siendo necesario mostrar las funcionalidades y el potencial de este tipo de herramientas, así como resolver los problemas técnicos más comunes que dificultan su uso (fundamentalmente en el caso de estudiantes de disciplinas no técnicas). Por otro lado, se recomienda su uso en fases tempranas de la formación universitaria, para suavizar la entrada en el modelo de educación superior, y en los últimos cursos del grado, para aumentar la compatibilidad entre los estudios y el trabajo. Como sea, la mera introducción de las herramientas de comunicación síncrona en la metodología docente no parece suficiente para involucrar a los alumnos desmotivados sino que será necesaria la adopción de otras técnicas innovadoras (p. ej. gamification), cuya valoración queda fuera del alcance de este artículo y se plantea como trabajo futuro.

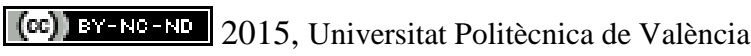

Congreso In-Red (2015) 
Disposición del alumnado al uso de herramientas de comunicación síncrona en la docencia universitaria

\section{Referencias}

ADOBE CONNECT (2015). <http://www.adobe.com/products/adobeconnect.html> [Consulta: 30 de mayo de 2015]

BIGBLUEBUTTON (2015). <http://bigbluebutton.org/> [Consulta: 30 de mayo de 2015]

BLACKBOARD COLLABORATE (2015). < http://www.blackboard.com> [Consulta: 30 de mayo de 2015]

BURNETT, C. (2003). “Learning to chat: Tutor participation in synchronous online chat” en Teaching in Higher Education, vol. 8, pp. 247-261.

DÍAZ, L. A., ESTEBAN, P. G., TOSINA, R. Y., MASA, J. A., CUBO, S., \& DOS REI, A. D. (2014). "Usos de aulas virtuales síncronas en educación superior". Pixel-Bit: Revista de medios y educación, (45), pp. 203-215.

DOTLRN (2015). <http://dotlrn.org/> [Consulta: 24 de mayo de 2015]

DÖHRMANN, M., KAISER, G. y BLÖMEKE, S. (2014). "The conceptualisation of mathematics competencies in the International Teacher Education Study TEDS-M”. En S. Blömeke, F. J. Hsieh, G. Kaiser y W. H. Schmidt (Eds.), International Perspectives on Teacher Knowledge, Beliefs and Opportunities to Learn (pp. 431-456). Dordrecht, The Netherlands: Springer.

GRIMALDO-MORENO, F., AREVALILLO-HERRÁEZ, y LÓPEZ-IÑESTA, E. (2011). "Utilización de una herramienta de comunicación online para la mejora docente. Dos casos prácticos” en Jornadas de Enseñanza Universitaria de la Informática (JENUI 2011), pp. 101-108.

GRIMALDO-MORENO, F. FUERTES-SEDER, A., PÉREZ-CONDE, C., AREVALILLOHERRÁEZ, M., COBOS-SERRANO, M. and MORENO-CLARÍ, P. (2014). "Big Blue Button, una alternativa de código abierto para la comunicación interactiva en actividades educativas” en Jornadas de Enseñanza Universitaria de la Informática (JENUI 2014), pp. 75-82.

GÓMEZ, P. y GUTIÉRREZ-GUTIÉRREZ, A. (2014). “Conocimiento matemático y conocimiento didáctico del futuro profesor español de primaria en Resultados del estudio TEDS-M”. González, M. T., Codes, M., Arnau, D. y Ortega, T. (coord.). En: Investigación en Educación Matemática XVIII. Salamanca: SEIEM, pp. 99-114

GOOGLE HANGOUTS (2015). <http://www.google.com/+/learnmore/hangouts/> [Consulta: 30 de mayo de 2015] 
Francisco Grimaldo, Emilia López-Iñesta, David Arnau, Máximo Cobos-Serrano, Ricardo Ferrís, Miguel García-Pineda, Juan Gutiérrez-Soto, Paloma Moreno-Clarí, Mario Zacarés-González y

Carlos Pérez-Condes

HSIEH, Y. H. y TSAI, C.-C. (2012). “The effect of moderator's facilitative strategies on online synchronous discussions” en Computers in Human Behavior, vol. 28, issue 5, pp. 1708-1716.

JOHNSON, G. M. (2005). "Student alienation, academic achievement, and WebCT use” en Educational Technology and Society, vol. 8, pp. 179-189.

JOHNSON, G. (2008). “The relative learning benefits of synchronous and asynchronous text-based discussion” en British Journal of Educational Technology, vol. 39, issue 1, pp. 166-169.

LINSTONE, H. A. y TUROFF, M. (1975). “Introducción” en Linstone, H. A. y Turoff, M. (Eds.). The Delphi method: Techniques and applications. Reading, MA: Addison-Wesley, pp. 3-12.

LOBEL, M., NEUBAUER, M., y SWEDBURG, R. (2002). "Elements of group interaction in a real-time synchronous online learning-by-doing classroom without F2F participation” en USDLA Journal, vol. 16, issue 4, pp. 20-22.

MOODLE, 2015, <https://moodle.org/> [Consulta: 24 de mayo de 2015]

OFFIR, B., LEV, Y., y BEZALEL, R. (2008). "Surface and deep learning processes in distance education: Synchronous versus asynchronous systems” en Computers \& Education, vol. 51, issue 3, pp. 1172-1183.

PEÑA, D. (2002). Análisis de datos multivariantes. Madrid: McGraw-Hill / Interamericana de España

SANCERNI, M.D. (2012). "Herramientas virtuales síncronas: rompiendo barreras en la universidad presencial” en Revista del CIDUI, vol. 1.

WANG, M. (2004). "Correlational Analysis of Student Visibility and Performance in Online Learning” en Journal of Asynchronous Learning Network, vol. 8, pp. 71-82.

Agradecimientos: Esta experiencia ha sido desarrollada en el marco de una Red de Innovación Educativa. Los autores agradecen la financiación recibida desde el Vicerrectorado de Convergencia Europea y Calidad de la Universitat de València, a través del proyecto Finestra Oberta con código UV-SFPIE_FO14-223160.

(c)) EY-NC-ND 2015, Universitat Politècnica de València

Congreso In-Red (2015) 
Disposición del alumnado al uso de herramientas de comunicación síncrona en la docencia universitaria

\section{Anexo I}

\section{CUESTIONARIO SOBRE EL USO DE HERRAMIENTAS DE COMUNICACIÓN POR} INTERNET COMO SOPORTE PARA LA DOCENCIA UNIVERSITARIA

Instrucciones: Con el fin de recoger su opinión en relación a la docencia de esta asignatura y la iniciativa de emplear herramientas para la comunicación por Internet (videoconferencias, chat, Skype, FaceTime, etc.) en la misma, se presenta el siguiente cuestionario sobre el que se solicita su colaboración.

Todos los datos serán tratados de manera anónima y confidencial con el fin de valorar la propuesta docente y realizar mejoras mediante futuras acciones.

Después de la información académica y personal solicitada, encontrará una serie de afirmaciones que deberán ser señaladas, indicando su grado de acuerdo. Gracias por su participación.

Información personal y académica

Género: $\square$ Masculino $\square$ Femenino

Edad: $\square 19$ o menos $\square$ 20-21 $\square$ 22-23 $\square$ 24-25 $\square$ Más de 25

Universidad:

Acceso a la titulación en la que se encuentra matriculado:

$\square$ PAU $\quad \square$ FP $\square$ Título Universitario $\square$ Otros

Asignatura en la que se encuentra matriculado:

Titulación en la que se encuentra matriculado: $\square$ Grado $\square$ Máster

Nombre:

Curso más alto en el que se está matriculado:
$1^{\circ} \square 2^{\circ}$
$\square 3^{\circ} \quad \square 4^{\circ}$

Curso más bajo en el que se está matriculado: Número de veces que se ha matriculado en la asignatura:

$\square 1^{\circ} \quad \square 2^{\circ} \quad \square 3^{\circ} \quad \square 4^{\circ}$

La asignatura me interesa:

$\square$ Nada $\square$ Algo $\square$ Bastante $\square$ Mucho

$\square 1 \square 2 \square 3 \quad \square$ Más de 3

Suelo hacer uso de las tutorías presenciales: $\square$ Nada $\quad \square$ Algo $\square$ Bastante $\quad \square$ Mucho

Compatibilizo estudios con trabajo: $\square \quad$ Calificación esperada: $\square$ NP $\square$ Sus $\square$ Ap

Sí $\square$ No

$\square$ Not $\square$ Sb $\square$ M.H.

Manejo de herramientas informáticas

\begin{tabular}{cccccc}
\hline & $\begin{array}{c}\text { Muy en } \\
\text { desacuerdo }\end{array}$ & $\begin{array}{c}2 . \\
\text { En } \\
\text { desacuerdo }\end{array}$ & $\begin{array}{c}3 . \\
\text { Indiferente }\end{array}$ & $\begin{array}{c}4 . \\
\text { De } \\
\text { acuerdo }\end{array}$ & $\begin{array}{c}\text { Muy de } \\
\text { acuerdo }\end{array}$ \\
\hline $\begin{array}{c}\text { Tengo un buen } \\
\text { manejo en general } \\
\text { del ordenador y sus } \\
\text { programas }\end{array}$ & $\square$ & $\square$ & $\square$ & $\square$ & $\square$ \\
\hline
\end{tabular}

(c)) EY-NC-ND 2015, Universitat Politècnica de València 
Francisco Grimaldo, Emilia López-Iñesta, David Arnau, Máximo Cobos-Serrano, Ricardo Ferrís, Miguel García-Pineda, Juan Gutiérrez-Soto, Paloma Moreno-Clarí, Mario Zacarés-González y

Carlos Pérez-Condes

\begin{tabular}{|c|c|c|c|c|c|}
\hline $\begin{array}{c}\text { Suelo tener } \\
\text { problemas con la } \\
\text { tecnología } \\
\end{array}$ & $\square$ & $\square$ & $\square$ & $\square$ & $\square$ \\
\hline $\begin{array}{l}\text { Utilizo herramientas } \\
\text { para comunicarme } \\
\text { por Internet (Skype, } \\
\text { Messenger, etc.) }\end{array}$ & $\square$ & $\square$ & $\square$ & $\square$ & $\square$ \\
\hline $\begin{array}{c}\text { Conozco el } \\
\text { funcionamiento de la } \\
\text { plataforma } \\
\text { docente/aula virtual }\end{array}$ & $\square$ & $\square$ & $\square$ & $\square$ & $\square$ \\
\hline
\end{tabular}

Frecuencia de uso de herramientas para la comunicación por Internet (Skype, Messenger, Facetime, etc.)

\begin{tabular}{cccccc}
\hline & $\begin{array}{c}1 . \\
\text { Nunca }\end{array}$ & $\begin{array}{c}2 . \\
\text { Rara vez }\end{array}$ & $\begin{array}{c}\text { 3. } \\
\text { Algunas } \\
\text { veces }\end{array}$ & $\begin{array}{c}4 . \\
\text { Casi } \\
\text { siempre }\end{array}$ & $\begin{array}{c}5 . \\
\text { Siempre }\end{array}$ \\
\hline En asignaturas de la titulación & $\square$ & $\square$ & $\square$ & $\square$ & $\square$ \\
\hline En cursos online & $\square$ & $\square$ & $\square$ & $\square$ & $\square$ \\
\hline Contacto con familia/amigos & $\square$ & $\square$ & $\square$ & $\square$ & $\square$ \\
\hline Trabajo & $\square$ & $\square$ & $\square$ & $\square$ & $\square$ \\
\hline $\begin{array}{c}\text { ¿Conoce alguna de estas } \\
\text { herramientas? }\end{array}$ & $\square$ & $\square$ & $\square$ & $\square$ & $\square$ \\
& Skype & $\begin{array}{c}\text { Black } \\
\text { board } \\
\text { Colla } \\
\text { borate }\end{array}$ & $\begin{array}{c}\text { BigBlueB } \\
\text { utton }\end{array}$ & $\begin{array}{c}\text { Adobe } \\
\text { Connect }\end{array}$ & Google \\
& & $\square$ & & \\
\hline
\end{tabular}

Indique el nombre de las herramientas que utiliza con más frecuencia para la comunicación por Internet

Por favor, escribir en este espacio con mayúsculas (puede escribir más de una opción):

Expectativas iniciales para el uso de las herramientas de comunicación por Internet en la asignatura

\begin{tabular}{cccccc}
\hline & $\begin{array}{c}\text { Muy en } \\
\text { desacuerdo }\end{array}$ & $\begin{array}{c}2 . \\
\text { En } \\
\text { desacuerdo }\end{array}$ & $\begin{array}{c}\text { 3. } \\
\text { Indiferente }\end{array}$ & $\begin{array}{c}4 . \\
\text { De } \\
\text { acuerdo }\end{array}$ & $\begin{array}{c}\text { Muy de } \\
\text { acuerdo }\end{array}$ \\
\hline $\begin{array}{c}\text { La iniciativa me parece } \\
\text { interesante }\end{array}$ & $\square$ & $\square$ & $\square$ & $\square$ & $\square$ \\
\hline $\begin{array}{c}\text { Me siento con mayor seguridad } \\
\text { para afrontar la asignatura }\end{array}$ & $\square$ & $\square$ & $\square$ & $\square$ & $\square$ \\
\hline $\begin{array}{c}\text { Es útil para mantener la } \\
\text { comunicación profesor/alumno }\end{array}$ & $\square$ & $\square$ & $\square$ & $\square$ & $\square$ \\
\hline Puede facilitar la resolución de dudas & $\square$ & $\square$ & $\square$ & $\square$ & $\square$ \\
\hline $\begin{array}{c}\text { Puedo ahorrar tiempo y evitar } \\
\text { desplazamientos }\end{array}$ & $\square$ & $\square$ & $\square$ & $\square$ & $\square$ \\
\hline Prefiero sesiones presenciales & $\square$ & $\square$ & $\square$ & $\square$ & $\square$ \\
\hline
\end{tabular}

(cc)) EY-NC-ND 2015, Universitat Politècnica de València

Congreso In-Red (2015) 